2. Akinosoglou K, Apostolakis E, Koutsogiannis N, Leivaditis V, Gogos CA. Rightsided infective endocarditis: surgical management. Eur J Cardiothorac Surg. 2012;42:470-9.
3. Slaughter MS, Badhwar V, Ising M, Ganzel BL, Sell-Dottin K, Jawitz OK, et al Optimum surgical treatment for tricuspid valve infective endocarditis: an analysis of the Society of Thoracic Surgeons national database. J Thorac Cardiovasc Surg. 2021;161:1227-35.e1.
See Article page 1227.

\section{Commentary: Endocarditis of the forgotten valve: Forget about valvectomy?}

\author{
Siamak Mohammadi, MD, FRCSC, and \\ Dimitri Kalavrouziotis, MD, FRCSC
}

The causes of tricuspid valve (TV) endocarditis are multiple, although intravenous drug use (IVDU) remains a leading factor. Parallel with the exponential rise in heroin-related deaths in North America, ${ }^{1}$ the incidence of TV endocarditis is increasing, ${ }^{2}$ and it is developing into an important societal problem. Few studies ${ }^{3,4}$ have investigated large numbers of IVDU patients undergoing TV surgery for endocarditis, and the outcomes of this surgery in the current era. The most recent peer-reviewed guidelines contribute little in terms of selecting the optimal surgical approach for IVDU patients with TV endocarditis. ${ }^{5}$

In this issue of the Journal, Slaughter and colleagues ${ }^{6}$ evaluate the incidence and early outcomes associated with first-time isolated TV surgery for endocarditis among IVDU patients in a large contemporaneous cohort using the Society of Thoracic Surgeons (STS) database. This retrospective study comprised 1613 IVDU patients with a median age of 30 years who underwent 1 of 3 different types of TV surgery: valvectomy $(n=119)$, repair $(n=532)$, or replacement $(n=962)$. The investigators found that the incidence of TV endocarditis operations due to IVDU increased significantly during the study period. As

\footnotetext{
From the Department of Cardiac Surgery, Quebec Heart and Lung Institute, Quebec City, Quebec, Canada.

Disclosures: Authors have nothing to disclose with regard to commercial support.

Received for publication Nov 13, 2019; revisions received Nov 13, 2019; accepted for publication Nov 14, 2019; available ahead of print Dec 7, 2019.

Address for reprints: Siamak Mohammadi, MD, FRCSC, Department of Cardiac Surgery, Quebec Heart and Lung Institute, 2725 Chemin Sainte-Foy, Quebec City, Quebec, Canada G1V 4G5 (E-mail: siamak.mohammadi@fmed.ulaval.ca).

J Thorac Cardiovasc Surg 2021;161:1237-8

$0022-5223 / \$ 36.00$

Copyright (C) 2019 by The American Association for Thoracic Surgery

https://doi.org/10.1016/j.jtcvs.2019.11.066
}

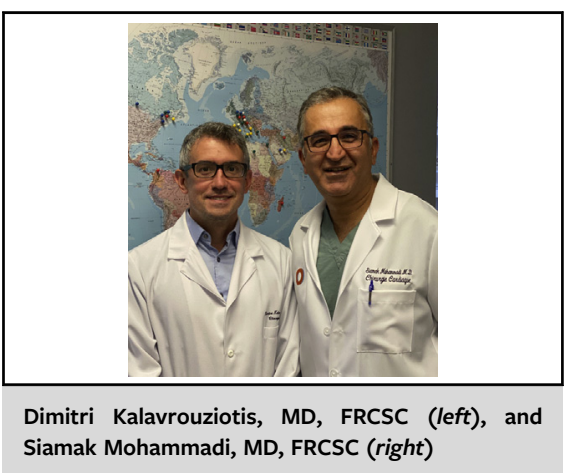

CENTRAL MESSAGE

Valve repair should be favored in drug-related tricuspid valve endocarditis. Valvectomy is associated with early death, but there may still be an indication for this approach in certain patient subsets.

expected, patients with TV valvectomy had many markers of higher operative risk at baseline, including a higher prevalence of active endocarditis, Staphylococcus aureus as the primary pathogen, and a higher MELD (Model for EndStage Liver Disease) score. Valvectomy was associated with a significantly higher unadjusted early mortality $(16 \%)$ compared with TV repair $(2 \%)$ or replacement $(3 \%)$. Multivariable analysis adjusted for meaningful covariates, including the acuity of infection, emergency surgery, and, importantly, TV surgery hospital volume, identified TV valvectomy as an independent risk factor for inhospital or 30-day mortality. Interestingly, the type of valve surgery had no effect on major adverse events, a composite of 7 different outcomes. This may reflect the fact that the higher mortality among valvectomy recipients provides a competing risk, such that these patients do not have the same opportunity to develop complications as patients after repair or replacement.

The authors concluded that TV valvectomy should be avoided in IVDU patients with TV endocarditis in favor 
of TV repair or replacement, both of which have similar early results. Emergency surgery, perioperative extracorporeal membrane oxygenation requirement, leukocytosis, and lower hospital TV procedural volume were the other independent risk factors associated with in-hospital or 30-day mortality.

The study is laudable because it is a collaborative effort and one of the largest series to date investigating the timely problem of IVDU-related endocarditis, which unfortunately continues to increase in industrialized nations and decimates global life expectancies as a problem of young people. What the use of the STS database allows for in terms of well-powered analyses over large expanses of time, it lacks in terms of data granularity, and several caveats related to this study must be mentioned. First, the decision-making process that leads a surgeon to select a strategy of TV valvectomy for a given patient is complex and most certainly depends on more than just "eyeballing" the valve and assessing the extent of tissue destruction. Such variables as lung abscess, empyema, and other active nontreated infections, as well as a perceived higher risk of IVDU recidivism, may influence the surgeon to choose valvectomy even if the extent of tissue destruction is compatible with a TV replacement.

These same variables, which are not captured by the STS database, are powerful drivers of mortality, possibly secondary to ongoing postoperative sepsis and multisystem organ failure. In the absence of uniformly accepted criteria for patient selection for each type of TV surgery, it is difficult to incriminate TV valvectomy as a leading cause of mortality in patients with IVDU-related TV endocarditis, as there is a residual selection bias favoring TV repair and replacement that cannot simply be adjusted out in multivariable analyses. In addition to the higher early mortality rate associated with valvectomy compared with repair and replacement, a significantly higher rate of infectious causes of death among the valvectomy subgroup suggests a greater role of extracardiac sources of infection (eg, lung abscess) as a major cause of early mortality in valvectomy recipients, despite having addressed the TV infection, and confirms a higher risk profile in these patients.

An important limitation of this study is the absence of longitudinal data. This is a major concern in IVDU patients compared with non-drug-related endocarditis, because of the young age of IVDU patients and the risk of recidivism and early and late reinfection. The risk of recurrent drug use, recurrent infection of the prosthetic TV materials, and subsequent risk of death remain important and unknown. Other relevant unknowns that were not addressed by Slaughter and colleagues include the optimal repair technique, whether a TV prosthetic ring should be avoided at all costs during repair, and the durability of repair, especially when there is preexisting severe TV insufficiency.

The results of this study underscore the increasing burden of IVDU-related TV endocarditis, which primarily affects young patients, and the urgent need to focus our efforts on improving early surgical outcomes and the risk of future reinfection in this population. ${ }^{5}$ The authors suggest that TV repair should be the surgical approach of choice in patients with IVDU endocarditis to achieve better early clinical outcomes. Most surgeons would agree with this for both IVDU-related and other causes of endocarditis. Increasing the feasibility of TV repair implies an earlier surgical approach to prevent extensive TV leaflet destruction and secondary septic pulmonary emboli. The authors' results suggest that a moratorium should be placed on TV valvectomy, as it was shown to be an independent predictor of mortality despite risk adjustment using the available covariates provided by the STS database. However, many surgeons with extensive expertise in TV endocarditis would likely disagree; the role of valvectomy in patients with an extremely high risk of reinfection and as a bridge to infection eradication and drug rehabilitation continues to be debated. ${ }^{7}$ Future studies should focus on the risk of early and late reinfection, as well as the risk of recidivism of intravenous drug use and the impact of dedicated rehabilitation strategies, to allow us to make informed decisions in this challenging area of cardiac surgery.

\section{References}

1. Centers for Disease Control and Prevention (CDC). Drug overdose deaths; . Available at: https://www.cdc.gov/drugoverdose/data/statedeaths.html. Accessed October 19, 2019.

2. Wallen TJ, Szeto W, Williams M, Atluri P, Arnaoutakis G, Fults M, et al. Tricuspid valve endocarditis in the era of the opioid epidemic. J Card Surg. 2018;33:260-4.

3. Gaca JG, Sheng S, Daneshmand M, Rankin JS, Williams ML, O'Brien SM, et al. Current outcomes for tricuspid valve infective endocarditis surgery in North America. Ann Thorac Surg. 2013;96:1374-81.

4. Dawood MY, Cheema FH, Ghoreishi M, Foster NW, Villanueva RM, Salenger R, et al. Contemporary outcomes of operations for tricuspid valve infective endocarditis. Ann Thorac Surg. 2015;99:539-46.

5. Pettersson GB, Coselli JS, Hussain ST, Griffin B, Blackstone EH, Gordon SM, et al. 2016 The American Association for Thoracic Surgery (AATS) consensus guidelines: Surgical treatment of infective endocarditis: executive summary. $J$ Thorac Cardiovasc Surg. 2017;153:1241-58.e29.

6. Slaughter MS, Badhwar V, Ising M, Ganzel BL, Sell-Dottin K, Jawitz OK, et al. Optimum surgical treatment for tricuspid valve infective endocarditis: an analysis of the Society of Thoracic Surgeons national database. J Thorac Cardiovasc Surg. 2021;161:1227-35.e1.

7. Protos AN, Trivedi JR, Whited WM, Rogers MP, Owolabi U, Grubb KJ, et al. Valvectomy versus replacement for the surgical treatment of tricuspid endocarditis. Ann Thorac Surg. 2018;106:664-9. 